\title{
Decision Model of Aero-Engine Design Integration Platform Implementation Solution Selection Based on Number Interval Number with Priority Order
}

\author{
Ying $\mathrm{He}^{1,2}$ and Rui Xiu ${ }^{3}$ \\ ${ }^{1}$ School of mechanical engineering and automation, Beihang University, Beijing, China \\ ${ }^{2}$ AECC Hunan Aviation Power Plant Research Institute, Zhuzhou, China \\ ${ }^{3}$ Beijing Institute of Aerospace Control Instrument, Beijing, China
}

\begin{abstract}
Considering the fuzzy factors in the aero-engine design integration platform implementation solution selection, using the prioritized interval number evaluation method, the implementation model of the aero-engine design integration platform implementation solution selection is established, and the priority relationship is calculated according to the relative distance between each attribute and the ideal point. Through the calculation of the relative distance between the interval value and the negative ideal point, the single evaluation value is obtained. Finally, the comparison result of the pre-selected scheme is obtained by analysing and solving the preferred model. The model comprehensively considers the determining factors in the optimization process and uncertain factors, clear logic, and the best results obtained are comprehensive and reasonable.
\end{abstract}

\section{Introduction}

The main functions of the aero-engine design integration platform are to integrate, package, and call aero-engine design tools, define and manage the aero-engine design process, manage the control relationships and data flows between design tasks, finally, the accumulation, inheritance and reuse of aero-engine design knowledge, concepts, norms and experiences will be realized. Aeroengine design integration platform implementation solution selection decision problem is a multiple attribute decision making problem. Multiple attribute decision making is also called finite-plan multi-objective decisionmaking [1]. It refers to the decision-making problem of selecting the optimal alternative or sorting the scheme when considering multiple attributes. It is the modern decision-making science. An important part. Its theory and methods are widely used in many fields such as engineering, technology, economics, management and military. The main problem solved by multi-attribute decision-making is in the aspects of evaluation and selection. There are common elements in multiple attribute decision making [2-3]: 1. Multiple options: Before making group decisions, decision makers must first measure the number of feasible solutions as an option for evaluation.2. Multiple evaluation attributes: Before making a group decision, the decision maker must first measure the number of feasible attributes and propose several related attributes that affect the plan. The attributes can be independent or related.3. Weight assignment of attributes: Different attribute decision makers have different preference tendencies, and different weights are assigned to different attributes. Generally, the weight distribution of attributes is usually normalized.

\section{Statement of aero-engine design integration platform implementation solution selection decision problem}

Aero-engine design integration platform implementation decision problem where the attribute values are represented by the intervals and priority ordering. The following formula can describe the decision problem in detail:

In a multi-attribute decision problem, the set of alternatives is $F=\left\{f_{1,} f_{2,} f_{3}, \ldots . ., f_{i}\right\}$, among them $i, i \geq 2$, the set of evaluation attributes considered is $U=\left\{u_{1}, u_{2}, u_{3}, \ldots . ., u_{j}\right\}$, among them $j, j \geq 2$. The decision matrix of the initial multi-attribute decision problem is:

$$
A=\left[\begin{array}{cccc}
a_{11} & a_{12} & \ldots & a_{1 j} \\
a_{12} & a_{22} & \ldots & a_{2 j} \\
\ldots & \ldots & \ldots & \ldots \\
a_{i 1} & a_{i 2} & \ldots & a_{i j}
\end{array}\right]
$$


Where $a_{m n}$ represents the initial decision indicator value of the $n$ attribute of the $m$ scheme, and the value may be a certain value or a fuzzy value, which may be quantitative or qualitative.

There are three main steps to solve the problem: the first step is to establish an attribute evaluation system, the second step is to determine the attribute weight, and the final step is to use the evaluation method to evaluate the alternative.

The method of attribute value normalization can be divided into two categories, one is the normalization method of deterministic attribute value, and the other is the normalization method of fuzzy attribute value. The canonical methods for determining the attribute values can be divided into linear transformation, extreme transformation, vector transformation, trigonometric transformation, etc. The principle is to distinguish between the benefit attribute and the cost type attribute. However, the normative methods of fuzzy attribute values are more class. For qualitatively characterized control variables, considering the incompleteness of information and the limitations of risk diagnosis expert knowledge, it is often difficult to express their original information with precise numbers, and fuzzy expression. Sometimes it is more conducive to risk diagnosis experts to express their preferences. The representations of fuzzy expression mainly include interval numbers, triangular fuzzy numbers, trapezoidal fuzzy numbers, intuitionistic fuzzy numbers, language scales, and binary semantics. In the decision-making process, although different fuzzy language representations and aggregation methods will be used to obtain different results, there are no advantages or disadvantages in terms of various fuzzy language representations.

\section{Aero-engine design integration platform implementation solution selection decision model}

In terms of the attribute $U_{j}$, Specify priority orderings or ordered vectors $X^{j}=\left[\begin{array}{lll}x^{j} & (1), \quad x^{j}(2), \quad \ldots, \quad x^{j}(m)\end{array}\right]$ for alternatives with a priority relationship [4-7]:

$$
q_{s t}^{j}=\frac{1}{2}+\frac{x^{j}(\mathrm{t})}{2(\mathrm{n}-1)}+\frac{x^{j}(s)}{2(\mathrm{n}-1)}, \quad s, t=1,2, \ldots, n
$$

In addition, the classification values for the alternatives can be retrieved as follows:

$$
\omega_{i}^{j}=\frac{1}{m(m-1)}\left(\sum_{k=1}^{m} p_{i k}^{j}+\frac{m}{2}-1\right), s, t=1,2, \ldots, n
$$

So the standardized attribute values of the alternatives $A_{j}$ are calculated relative to the attributes as follows [8]:

$$
b_{i j}=\frac{\left|\omega_{i}^{j}-\min _{1 \leq k \leq m}\left\{\omega_{k}^{j}\right\}\right|}{\left|\omega_{i}^{j}-\min _{1 \leq k \leq m}\left\{\omega_{k}^{j}\right\}\right|+\left|\omega_{i}^{j}-\max _{1 \leq k \leq m}\left\{\omega_{k}^{j}\right\}\right|}, \begin{gathered}
i=1,2, \ldots, n \\
j=1,2, \ldots, n
\end{gathered}
$$

Attribute value of range $\bar{a}_{i j}=\left(\left[a_{i j}^{L}, a_{i j}^{U}\right]\right)_{m \times 1}$, $i=1,2, \ldots, n$

$j=1,2, \ldots, n$, standardized using the following formulas:

$$
\begin{gathered}
\left\{\begin{array}{l}
q_{i j}^{L}=a_{i j}^{L} / \sum_{l=1}^{m} a_{l j}^{U}, \\
q_{i j}^{U}=a_{i j}^{U} / \sum_{l=1}^{m} a_{l j}^{L} \quad{ }_{\text {if }} C_{j} \text { it is used }
\end{array}\right. \\
\left\{\begin{array}{l}
q_{i j}^{L}=\left(1 / a_{l j}^{U}\right) / \sum_{l=1}^{m}\left(1 / a_{l j}^{L}\right) \\
q_{i j}^{U}=\left(1 / a_{l j}^{L}\right) / \sum_{l=1}^{m}\left(1 / a_{l j}^{U}\right) \\
\text { if } A_{j} \text { it is for cost }
\end{array}\right.
\end{gathered}
$$

Definition 3. The distance between the two attribute values is calculated as following formula:

$$
d\left(\widetilde{q}_{i j}, \widetilde{q}_{k j}\right)=\frac{\sqrt{2}}{2} \sqrt{\left(q_{i j}^{L}-q_{k j}^{L}\right)^{2}+\left(q_{i j}^{U}-q_{k j}^{U}\right)^{2}} \quad i=1,2, \ldots, m,
$$

It's easy to get $d\left(\widetilde{q}_{i j}, \widetilde{q}_{k j}\right) \geq 0$.

Definition 4. In terms of attributes $A_{j}$, positive ideal values $\tilde{q}_{i j}=\left[q_{i j}^{L}, q_{i j}^{U}\right]\left(\begin{array}{c}i=1,2, \ldots, n \\ j=1,2, \ldots, n\end{array}\right)$ are defined as follows:

$$
\text { ideal }_{j}^{+}=\left[\text {ideal }_{j}^{+L}, \text { ideal }_{j}^{+U}\right]
$$

Over there,

$$
\begin{gathered}
\text { ideal }_{j}^{+L}=\max _{1 \leq i \leq m}\left\{q_{i j}^{L}\right\}, \quad j=1,2, \ldots, n \\
\text { ideal }_{j}^{+U}=\max _{1 \leq i \leq m}\left\{q_{i j}^{U}\right\}, \quad j=1,2, \ldots, n
\end{gathered}
$$

Definition 5. Reference attribute $A_{j} \quad j=1,2, \ldots, n$, the negative ideal attribute value of the attribute $A_{j}, j=1,2, \ldots, n$, are defined as follows:

$$
n e g_{j}^{-}=\left[n e g_{j}^{-L}, n e g_{j}^{-U}\right], j=1,2, \ldots, n
$$


Over there,

$$
\begin{aligned}
n e g_{j}^{-L} & =\min _{1 \leq i \leq m}\left\{a_{i j}^{L}\right\}, \quad j=1,2, \ldots, n \\
n e g_{j}^{-U} & =\min _{1 \leq i \leq m}\left\{a_{i j}^{U}\right\}, \quad j=1,2, \ldots, n
\end{aligned}
$$

Definition 6. Attribute $A_{j} \quad j=1,2, \ldots, n$ is the relative distance between the range attribute value $\widetilde{a}_{i j}=\left[a_{i j}^{L}, a_{i j}^{U}\right]$ and negative ideal value ${ }^{n e g_{j}^{-}}$are defined as follows:

For attribute $A_{j} \quad j=1,2, \ldots, n$, the relative distance between the attribute values of intervals $\tilde{a}_{i j}=\left[a_{i j}^{L}, a_{i j}^{U}\right]$ and the negative ideal one ${ }^{n e g_{j}^{-}}$is defined as:

$$
b_{i j}=\frac{d\left(\widetilde{q}_{i j}, n e g_{j}^{-}\right)}{d\left(\widetilde{q}_{i j}, \operatorname{pos}_{j}^{+}\right)+d\left(\widetilde{q}_{i j}, n e g_{j}^{-}\right)}, \quad j=1,2, \ldots, n
$$

Therefore, the attribute values $\widetilde{q}_{i j}$ of $A_{j} \quad j=1,2, \ldots, n$ will be standardized, especially in the middle $b_{i j} \quad i=1,2, \ldots, n \quad j=1,2, \ldots, n$.

On the basis of the processed matrix $\left.\mathrm{B}={ }^{\left(b_{i j}\right.}\right)_{m \times n}$, the following plan model is used to determine the attribute weights to maximize the balance of all options:

$$
\begin{gathered}
\max \sum_{i=1}^{m} \sum_{j=1}^{n} b_{i j} w_{j} \\
\sum_{j=1}^{n} w_{j}^{2}=1
\end{gathered}
$$

$0 \leq w_{j} \leq 1, j=1,2, \ldots, n$

The best solution for model (11-1)-(11-2) is as follows:

$$
w_{j}^{*}=\left(\sum_{i=1}^{m} b_{i j}\right) / \sqrt{\sum_{j=1}^{n}\left(\sum_{i=1}^{m} b_{i j}\right)^{2}}
$$

List the following equations:

$$
L(W, \lambda)=\sum_{i=1}^{m} \sum_{j=1}^{n} b_{i j} w_{j}+\frac{\lambda}{2}\left(\sum_{j=1}^{n} w_{j}^{2}-1\right)_{j=1,2, \ldots, n}
$$

$\lambda$ represents Lagrangian multiplier here.

If $\partial L / \partial w_{\mathrm{j}}=0, \partial L / \partial \lambda=0$, the following result can be obtained:

$$
\begin{gathered}
\sum_{i=1}^{m} b_{i j}+\lambda w_{j}=0, \quad j=1,2, \ldots, n \\
\sum_{j=1}^{n} w_{j}^{2}-1=0 \quad j=1,2, \ldots, n
\end{gathered}
$$

Calculate the two formulas (14-1) and (14-2), it can be obtained:

$$
\begin{gathered}
\lambda=-\sqrt{\sum_{j=1}^{N}\left(\sum_{i=1}^{M} b_{i j}\right)^{2}} \quad j=1,2, \ldots, n \\
w_{j}^{*}=\sum_{i=1}^{m} b_{i j} / \sqrt{\sum_{j=1}^{n}\left(\sum_{i=1}^{m} b_{i j}\right)^{2}} \quad j=1,2, \ldots, n
\end{gathered}
$$

Also, for $w_{j}^{*}, j=1,2, \ldots, n$ their sum must be standardized, the total value of the alternatives you get based on the simple addition weighting way:

$$
T=\sum_{j=1}^{n} b_{i j} w_{j} \quad j=1,2, \ldots, n
$$

According to the values, the solution can be arranged in descending order.

\section{Solution of aero-engine design integration platform implementation solution selection decision problem}

Aero-engine design integration platform implementation decision problem is a typical multi-attribute decision problem with four attributes and five alternatives. According to the algorithm of the third part, the specific data of the decision matrix is obtained as follows:

$$
A_{1}=\left[\begin{array}{cccc}
1 & 3 & {[2.9,3.3]} & {[10.7,12.2]} \\
2 & 1 & {[3.4,3.9]} & {[9.9,11.1]} \\
3 & 2 & {[3.7,4.2]} & {[11.3,12.9]} \\
4 & 4 & {[2.7,3.2]} & {[12.5,14.3]} \\
5 & 5 & {[3.1,3.5]} & {[10.6,11.8]}
\end{array}\right]
$$

After normalizing the decision matrix, the standardized matrix shown below is obtained.

$$
A_{2}=\left[\begin{array}{cccc}
1 & 0.431 & 0.231 & 0.326 \\
0.722 & 1 & 0.801 & 0.694 \\
0 & 0.691 & 1 & 0.330 \\
0.278 & 0.003 & 0.043 & 0.478 \\
0.691 & 0.098 & 0.501 & 1
\end{array}\right]
$$


Applying the third part of the model to solve the problem, the results of the weight values of the attributes are shown in the following table:

Table 1. Weights value of attribute.

\begin{tabular}{ccccc}
\hline & $F_{1}$ & $F_{2}$ & $F_{3}$ & $F_{4}$ \\
\hline $\begin{array}{c}\text { Weights } \\
\text { Value }\end{array}$ & 0.2123 & 0.2611 & 0.2510 & 0.2819 \\
\hline
\end{tabular}

Use the third part of the formula to solve the $\mathrm{T}$ value, and then calculate the order of the program:

Table 2. T value solution to get the order.

\begin{tabular}{lccccc}
\hline & $U_{1}$ & $U_{2}$ & $U_{3}$ & $U_{4}$ & $U_{5}$ \\
\hline $\mathrm{T}$ & 0.960 & 0.297 & 0.788 & 0.523 & 0.319 \\
\hline Sorted & \multicolumn{5}{c}{$U_{1} \succ_{U_{3}} \succ U_{4} \succ_{U_{5}} \succ_{U_{2}}$} \\
\hline
\end{tabular}

\section{References}

1. Hwang C L, Yoon K. Multiple Attribute Decision Making [M]. Fuzzy multiple objective decision making :. Springer-Verlag, 1994: 1-531.

2. Ribeiro R A. Fuzzy multiple attribute decision making: A review and new preference elicitation techniques [J]. Fuzzy Sets \& Systems, 1996, 78 (2): 155-181.

3. Yang T, Hung C C. Multiple-attribute decision making methods for plant layout design problem $[\mathrm{J}]$. Robotics and Computer-Integrated Manufacturing, 2007, 23 (1): 126-137.

4. Wei G. Hesitant fuzzy prioritized operators and their application to multiple attribute decision making $[\mathrm{J}]$. Knowledge-Based Systems, 2012, 31 (7): 176-182.

5. Chen S J J, Krelle W. Fuzzy Multiple Attribute Decision Making: Methods and Applications [M]. Springer-Verlag New York, Inc. 1992.

6. Liu W W, Shi C S, Zhao S B. Dynamic comprehensive evaluation model with the feature of speed $[\mathrm{J}]$. Systems Engineering-Theory \& Practice, 2013.

7. Wei C, Liu P, Chang C. Failure mode and effects analysis using grey theory $[\mathrm{J}]$. Integrated Manufacturing Systems, 1990, volume 12 (3): 211$216(6)$

8. Wang Y M, Parkan C. Multiple attribute decision making based on fuzzy preference information on alternatives: Ranking and weighting $[\mathrm{J}]$. Fuzzy Sets \& Systems, 2005, 153 (3): 331-346. 